\title{
Análise da Precipitação e sua Relação com Sistemas Meteorológicos em Seropédica, Rio de Janeiro
}

\author{
José Francisco Oliveira Júnior ${ }^{1}$, Rafael Coll Delgado ${ }^{1}$, Givanildo Gois ${ }^{1}$, \\ Anne Lannes ${ }^{2}$, Flavia Oliveira Dias ${ }^{2}$, Jessica Cristina Souza ${ }^{2}$, Manuella Souza $^{2}$ \\ ${ }^{1}$ Departamento de Ciências Ambientais - DCA, Universidade Federal Rural do Rio de Janeiro - UFRRJ, \\ Seropédica/RJ, Brasil \\ ${ }^{2}$ Colégio Técnico da UFRRJ - CTUR, Seropédica/RJ, Brasil
}

\begin{abstract}
RESUMO
Dados pluviométricos horários da Estação Meteorológica Automática (EMA) foram preenchidos com dados da normal climatológica do INMET e com os dados do satélite TRMM via produto 3B43. Esses dois conjuntos preencheram as falhas e homogeneizaram os dados do período de 2000-2012, e foram comparados com a ocorrência dos sistemas sinóticos (ZCAS, ZCOU e SF) e com os episódios de El Niño e La Niña, em Seropédica-RJ. A avaliação foi dividida em escalas médias, percentuais sazonais e acumulados anuais. Os dados sinóticos foram obtidos da Climanálise (1996-2012). As características médias mostraram alta variabilidade interanual e intrassazonal dos sistemas sinóticos, seguida da interação dos sistemas de mesoescala e locais. Os meses com maior precipitação $(>120 \mathrm{~mm}$ ) foram dezembro, janeiro e março, e os meses com menor precipitação $(<60 \mathrm{~mm})$ foram maio, junho, julho e agosto. O verão (42\%) e inverno (10\%) possuem características bem definidas e distintas em seus padrões de chuva.
\end{abstract}

Palavras-chave: homogeneidade, El Niño - Oscilação Sul (ENOS), sistemas sinóticos, preenchimento de dados, Baixada Fluminense.

\section{Precipitation Analysis and its Relation to Meteorological Systems in Seropédica, Rio de Janeiro State}

\begin{abstract}
Hourly rainfall data from an Automatic Weather Station (AWS) were filled with normal climatology data provided by the National Institute of Meteorology (INMET) and TRMM satellite data via $3 \mathrm{~B} 43$ product. These two sets filled the gaps and homogenized data from the 2000-2012 period at the Agricultural Ecology station; they were then compared with precipitation occurrence of synoptic systems (SACZ, MCZ and FS) and El Niño and La Niña episodes in the municipality of Seropédica. Evaluation was divided into the following precipitation ranges: average, seasonal percentage, and annual cumulative. Synoptic data from the 1996-2012 period were obtained from 'Climanálise'. The average characteristics identified showed high interannual and intraseasonal variability of synoptic systems, followed by interaction of mesoscale and local systems. December, January and March showed the highest rainfall levels (>120 mm), while May, June, July and August presented the lowest rainfall levels $(<60 \mathrm{~mm})$. Summer $(42 \%)$ and winter $(10 \%)$ seasons have well-defined characteristics and are distinct in their rainfall patterns.
\end{abstract}

Keywords: homogeneity, El Niño Sothern Oscillation (ENSO), synoptic systems, data filling, Baixada Fluminense. 


\section{INTRODUÇÃO}

A precipitação pluvial é amplamente reconhecida por muitos pesquisadores e estudiosos como a variável climatológica mais importante na Região Tropical. Por sua tamanha importância nesta região, diversos estudos (Lyra et al., 2006; André et al., 2008; Dereczynski et al., 2009; Reboita et al., 2010; OliveiraJúnior et al., 2012; Wanderley et al., 2013) e métodos para sua caracterização foram desenvolvidos, para entender seus impactos e sua evolução no tempo, sua intensidade, as tendências, sua duração e a frequência na região.

A precipitação pluvial também é importante em razão de apresentar uma distribuição espacial e temporal que influencia drasticamente diversas atividades humanas, como, por exemplo, comércio, indústria, turismo, práticas agrícolas, defesa civil e planejamento urbano (morfologia urbana) (Dereczynski et al., 2009). Muitos estudos observacionais voltados para a caracterização do regime de chuva foram realizados na Região Metropolitana do Rio de Janeiro (RMRJ) (Moraes et al., 2005; André et al., 2008; Dereczynski et al., 2009; Reboita et al., 2010).

No entanto, poucos estudos foram realizados na região da Baixada Fluminense (Jourdan, 2007; Pereira \& Menezes, 2004; André et al., 2008), envolvendo a caracterização média dos elementos meteorológicos, em particular a precipitação pluvial, principalmente no município de Seropédica.

Apesar de sua grande importância em nível regional e global, como já mencionado anteriormente, existe uma carência destes dados pluviométricos principalmente pela falta de estações de superfície e pela exigência de que os equipamentos medidores (pluviômetros) e registradores (pluviógrafos) estejam em boas condições de funcionamento. Os pluviômetros e pluviógrafos espalhados pela superfície terrestre coletam informações da precipitação. No entanto, estas são válidas somente para densa área de estações localizada no entorno da área de estudo. Segundo Angelis et al. (2004), em um estudo para América do Sul (AS), o monitoramento detalhado sobre extensas áreas exige a existência de uma densa rede de pluviômetros, o que, em certos casos, é inviabilizado em regiões de difícil acesso, como florestas e regiões montanhosas. Tendo em vista essa dificuldade, estimativas de precipitação via satélite vêm se tornando uma alternativa promissora, para contribuir com um aprimoramento no conhecimento do regime da precipitação, através de uma análise mais detalhada, sobre a região.

Um avanço na área de Sensoriamento Remoto (SR) em nível orbital é o satélite Tropical Rainfall Measuring Mission (TRMM), destacando-se no fornecimento de dados da precipitação nas regiões intertropicais do planeta (Huffman et al., 2007). O satélite TRMM é um projeto de parceria entre a National Aeronautics and Space Administration (NASA) e a Japan Aerospace Exploration Agency (JAXA), e foi lançado em 27 de novembro de 1997, com o objetivo de monitorar e estudar a precipitação nos trópicos e subtrópicos (Kummerow et al., 1998, 2000), além de verificar a influência do clima global. É uma boa alternativa no monitoramento da precipitação em nível regional e em nível global, devido à carência de uma rede pluviométrica consistente temporal e espacialmente.

A caracterização dos padrões espaciais e temporais da precipitação pluvial, e a identificação dos sistemas meteorológicos responsáveis por esses padrões são de suma importância. Para isso, são utilizadas técnicas estatísticas (análise de componentes principais - ACP, análise de agrupamento, séries de Fourier, análise harmônica, distribuições de probabilidade e técnica de wavelet), ferramentas de SIG (Sistema Informação Geográfica), Sensoriamento Remoto (SR) e Modelagem Atmosférica (MA) para auxiliar nas análises meteorológicas e climáticas (Lyra et al., 2006; André et al., 2008; Zeri et al., 2011; OliveiraJúnior et al., 2012; Wanderley et al., 2013).

Os principais sistemas meteorológicos que atuam na RMRJ variam da escala sinótica à local e são classificados como produtores e inibidores de chuva. São estes: os Sistemas Frontais (SF), os Sistemas Convectivos de Mesoescala (SCM), a Zona de Convergência do Atlântico Sul (ZCAS), a Alta Subtropical do Atlântico Sul (ASAS), os Bloqueios Atmosféricos (BA), os sistemas de brisas (vale/montanha, lacustre e marítima/ terrestre), a precipitação orográfica, as tempestades convectivas, entre outros (Satyamurty et al., 1998; Dereczynski et al., 2009; Cataldi et al., 2010). 
Atualmente, o Grupo de Previsão de Tempo (GPT) sugere a definição da Zona de Convergência de Umidade (ZCOU), quando se configurarem as seguintes condições meteorológicas: (i) Similar à ZCAS, porém com duração de apenas três dias, sendo que, a partir do quarto dia, já é caracterizada como ZCAS; (ii) Quando a ZCAS começa a se dissipar, porém ainda pode ser identificada uma banda de nuvens organizada; neste último caso, nota-se que a convergência em $850 \mathrm{hPa}$ apresenta duas áreas preferenciais: uma em direção à ZCAS e outra em direção ao norte da Argentina, ao Paraguai e ao oeste da Região Sul do Brasil, mediante a presença do Jato de Baixos Níveis (JBN). Este último padrão pode ser associado à aproximação de uma frente ou de um cavado na média troposfera. Em determinados episódios, podem ocorrer simultaneamente as duas regiões de convergência de umidade, sendo que a direcionada para o sul do Brasil ou para a Argentina não gera nebulosidade. Essa situação coincide com a transição para a ZCOU (Climanálise, 2012).

Estes sistemas provocam precipitação de intensidade variável, dependendo da localização e da topografia da região, ou ainda inibem ou provocam fortes estiagens e veranicos no Estado do Rio de Janeiro. A presença dos Maciços de Pedra Branca, Tijuca e Gericinó, que compõem a RMRJ, oferece uma barreira ao deslocamento de ar nos níveis mais baixos da atmosfera e resultam em modificações na estrutura da circulação e nas próprias condições de tempo local e/ou em regiões adjacentes, juntamente com as Baías de Sepetiba e Guanabara, que interferem nos regimes de precipitação pluvial (Moraes et al., 2005). Os padrões sazonais e anuais dos sistemas meteorológicos que atuam na RMRJ podem ser influenciados por alguns modos de variabilidade climática, como o El Niño - a Oscilação Sul (ENOS) e a Oscilação Decadal do Pacífico (ODP) (Cataldi et al., 2010).

Baseado no exposto, o objetivo do trabalho consiste na comparação entre a precipitação observada e preenchida com os dados da normal climatológica, e os dados da precipitação observada e preenchida com os dados do TRMM, em várias escalas temporais, e sua relação com sistemas meteorológicos produtores de chuva no período de 2000-2012, em Seropédica, Rio de Janeiro.

\section{MATERIAL E MÉTODOS}

\section{1. Área de estudo}

O município de Seropédica, situado a $22^{\circ} 44^{\prime} 38^{\prime \prime} \mathrm{S}$ de latitude, $43^{\circ} 42^{\prime} 27^{\prime \prime}$ W de longitude e $26 \mathrm{~m}$ de altitude, pertence ao Estado do Rio de Janeiro (Figura 1) e tem área de aproximadamente $266,55 \mathrm{~km}^{2}$. Embora considerado politicamente

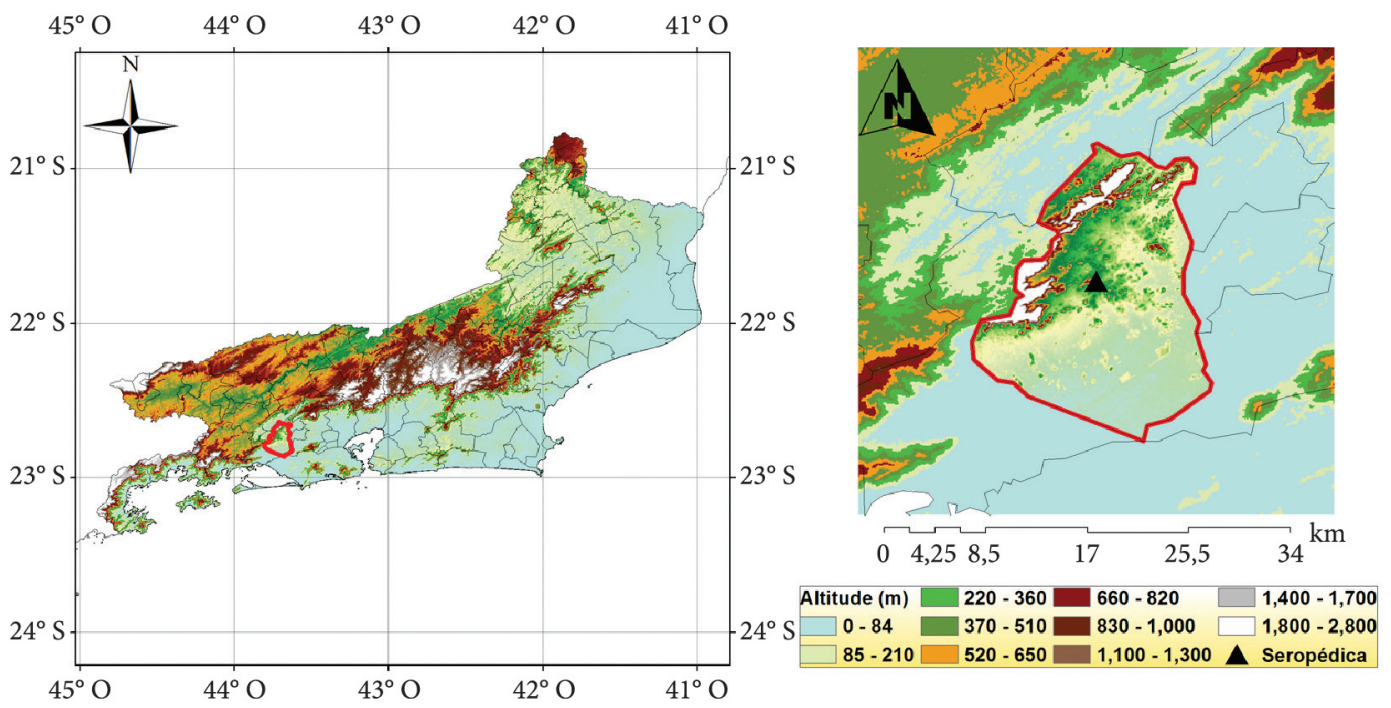

Figura 1. Mapa altimétrico ( $\mathrm{m}$ ) do Estado do Rio de Janeiro, destacando a altimetria (m) do município de Seropédica. Figure 1. Altimetry map (m) of Rio de Janeiro State highlighting altimetry $(\mathrm{m})$ Seropédica municipality. 
como integrante da RMRJ, geograficamente está situado em uma área da planície costeira fluminense denominada Baixada de Sepetiba. Limitada ao Sul pelo Oceano Atlântico, esta baixada possui como limites interiores a Serra do Mar a oeste (W) e noroeste (NW), o maciço da Pedra Branca a leste (E), a Serra dos Órgãos a nordeste (NE), a Serra da Mantiqueira a noroeste $(\mathrm{NW})$ e, ao norte $(\mathrm{N})$ e ao nordeste (NE), uma sucessão de morros de pequena altitude, que se incorporam à chamada paisagem de Mar de Morros (Gasparin et al., 2013). Segundo a classificação de Köppen, o clima da região é do tipo 'Aw', com chuvas concentradas entre novembro e março, precipitação anual média de $1.213 \mathrm{~mm}$ e temperatura média anual de $23,9^{\circ} \mathrm{C}$ (Carvalho et al., 2011).

\subsection{Séries de dados meteorológicos}

Foram coletados e organizados dados de precipitação horários ( $\mathrm{mm}$ ) da Estação Meteorológica Automática de Superfície (EMAS) de Seropédica, denominada de Ecologia Agrícola (EA), situada a $22^{\circ} 45^{\prime} 28^{\prime \prime} \mathrm{S}$ de latitude, $43^{\circ} 41^{\prime} 5^{\prime \prime} \mathrm{W}$ de longitude e $34 \mathrm{~m}$ de altitude, no período de 2000 a 2012, e estes foram convertidos em dados acumulados mensais. Os dados foram cedidos gentilmente pelo Instituto Nacional de Meteorologia (INMET).

Os dados do satélite TRMM de precipitação do produto 3B43 (http://mirador.gsfc.nasa.gov/cgibin/mirador/presentNavigation.pl?), com resolução espacial de aproximadamente $30 \mathrm{~km}$ e resolução temporal mensal, foram convertidos para valores mensais e anuais de 2000 a 2012, sendo obtidos no seguinte endereço eletrônico: http.www.mirador. gsfc.nasa.gov/collections/TRMM_3B43_007.shtml (TRMM, 2013).

O produto 3B43 é obtido no formato '.nc' (NetCDF) e, por isso, foi utilizado o programa ArcGIS 10.1 para a conversão e a leitura em planilhas. No software ArcGIS 10.1, foi utilizado o ArcToolbox - Multidimension Tools, e as ferramentas de conversão Make NetCDF Raster Layer e Make NetCDF Table View. A partir da conversão, foi possível escolher quatro pontos georreferenciados que fossem mais próximos do município de Seropédica e, consequentemente, da EMA (Figura 2).

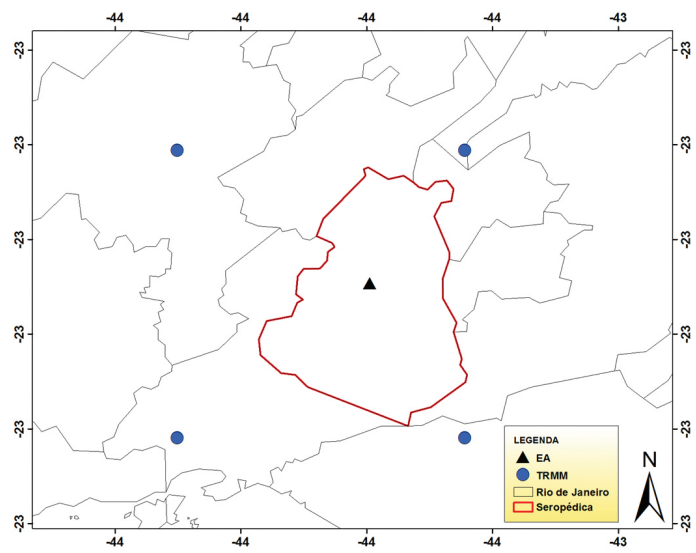

Figura 2. Distribuição espacial dos pontos escolhidos do TRMM para serem correlacionados ao ponto da EMA em Seropédica.

Figure 2. Spatial distribution of TRMM chosen points to be correlated to EMA location in Seropédica.

Os dados do satélite TRMM foram usados para preencher os dados de precipitação observados na EMAS/INMET e comparados com os dados preenchidos com a Normal Climatológica (NC) nos dados horários da EMAS/INMET de Seropédica, para posteriormente serem correlacionados e validados. Ambos os conjuntos podem servir para o preenchimento de falhas e homogeneização dos dados, pois existe um déficit de observações de superfície na região da Baixada Fluminense.

$\mathrm{Na}$ avaliação do produto 3B43 para determinar a precipitação em Seropédica, foram utilizados alguns métodos estatísticos baseados em análises comparativas entre as metodologias propostas, com base nos valores observados na EMAS e da normal climatológica proveniente do INMET, existentes para o município. Para validação dos modelos, foram adotados os seguintes métodos estatísticos: Regressão Linear e Correlação Linear (Pearson) $\left(r^{2}\right.$ e r), Ajustamento de Curvas, seguido do Erro Padrão de Estimativa (EPE) proposto por Allen et al. (1989), e o índice de concordância (d) proposto por Willmott et al. (1985). As formulações usadas seguem abaixo:

$r^{2}=\frac{\sum_{i=1}^{n}\left(P_{i}-\bar{O}\right)^{2}}{\sum_{i=1}^{n}\left(O_{i}-\bar{O}\right)^{2}}$ 


$$
r=\frac{\sum O_{i} P_{i}}{\sqrt{\sum O_{i}^{2} \sqrt{\sum P_{i}^{2}}}}
$$

$$
E P E=\sqrt{\frac{\sum\left(O_{i}-P_{i}\right)^{2}}{n-1}}
$$

$$
d=1-\left[\frac{\sum_{i=1}^{N}\left(P_{i}-O_{i}\right)^{2}}{\sum_{i=1}^{N}\left(\left|P_{i}-O_{i}\right|+\left|O_{i}-\bar{O}\right|\right)^{2}}\right]
$$

Em que:

- $\mathrm{Pi}=$ o iésimo valor estimado de precipitação ( $\mathrm{mm})$;

- Oi = o iésimo valor observado de precipitação $(\mathrm{mm})$;

- $\overline{\mathrm{O}}=$ valor médio observado de precipitação $(\mathrm{mm})$;

- $\mathrm{N}$ = número de dados analisados.

A partir disso, foram feitas avaliações médias, sazonal (verão - Dezembro, Janeiro e Fevereiro - DJF; outono - Março, Abril e Maio - MAM; inverno - Junho, Julho e Agosto - JJA, e primavera - Setembro, Outubro e Novembro - SON - neste artigo, será adotada esta nomenclatura ao longo do texto) e anual; e, por fim, essas avaliações foram associadas aos principais sistemas produtores de chuva (SFs, ZCAS e ZCOU) e aos eventos de ENOS (El Niño e La Niña).

Dados da Climanálise da atuação dos SFs, ZCAS e ZCOU no Estado do Rio de Janeiro foram utilizados no período de 1996-2012. Vale ressaltar que o estudo foi restrito à ocorrência desses sistemas sinóticos, pois estes ocorrem com maior frequência em comparação aos demais sistemas que atuam na região (Oliveira, 1986; Oliveira-Júnior, 2008; Dereczynski et al., 2009; Reboita et al., 2010). Os dados foram extraídos com auxílio do diagrama de frequência de ocorrência dos sistemas sinóticos na região e nos relatos dos boletins da Climanálise (2012). Após a contabilização dos totais anuais (valores absolutos) e percentuais (\%) (Tabela 1) de ocorrência, estes foram comparados com os eventos de ENOS (Tabela 2), a fim de verificar a influência destes eventos na variabilidade interanual desses sistemas no município de Seropédica-RJ. Para os eventos de El Niño e La Niña, foi considerada a região do El Niño $3.4\left(5^{\circ} \mathrm{N}-5^{\circ} \mathrm{S}, 120^{\circ} \mathrm{W}-170^{\circ} \mathrm{W}\right)$ (Tremberth, 1997).
Tabela 1. Totais anuais e frequência relativa (\%) dos episódios de ZCAS/ZCOU e atuação dos SFs no Estado do Rio de Janeiro, no período de 1996-2012.

Table 1. Annual total and relative frequency (\%) of SACZ/ZCOU episodes and SF's performance over Rio de Janeiro state at 1996-2012 period.

\begin{tabular}{|ccccc|}
\hline Anos & $\begin{array}{c}\text { Sistemas } \\
\text { Frontais }\end{array}$ & $\begin{array}{c}\text { Frequência } \\
(\%)\end{array}$ & $\begin{array}{c}\text { ZCAS/ } \\
\text { ZCOU }\end{array}$ & $\begin{array}{c}\text { Frequência } \\
(\%)\end{array}$ \\
\hline 1996 & 35 & 6 & 6 & 6 \\
\hline 1997 & 37 & 6 & 5 & 4 \\
\hline 1998 & 54 & 9 & 2 & 2 \\
\hline 1999 & 46 & 8 & 6 & 5 \\
\hline 2000 & 51 & 9 & 5 & 4 \\
\hline 2001 & 31 & 5 & 5 & 4 \\
\hline 2002 & 34 & 6 & 4 & 3 \\
\hline 2003 & 45 & 8 & 3 & 3 \\
\hline 2004 & 48 & 8 & 8 & 7 \\
\hline 2005 & 46 & 8 & 9 & 8 \\
\hline 2006 & 32 & 6 & 10 & 9 \\
\hline 2007 & 34 & 6 & 11 & 10 \\
\hline 2008 & 28 & 5 & 14 & 12 \\
\hline 2009 & 22 & 4 & 8 & 7 \\
\hline 2010 & 18 & 3 & 6 & 5 \\
\hline 2011 & 19 & 3 & 13 & 11 \\
\hline 2012 & 10 & 2 & 5 & 5 \\
\hline
\end{tabular}

Fonte: Climanálise (http://www6.cptec.inpe.br/revclima/ boletim/). Os dados de 2012 estão incompletos com relação à contabilização dos SF e ZCAS/ZCOU.

Tabela 2. Classificação dos tipos de ENOS, período e intensidade de 1997-2012.

Table 2. ENSO classification: types, intensity and period during 1997-2012.

\begin{tabular}{|ccc|}
\hline Período & Tipo de ENOS & Intensidade \\
\hline $1997-1998$ & El Niño & Forte \\
\hline $1998-1999$ & La Niña & Forte \\
\hline $2000-2001$ & La Niña & Fraca \\
\hline $2002-2003$ & El Niño & Moderado \\
\hline $2004-2005$ & El Niño & Fraco \\
\hline $2006-2007$ & El Niño & Fraco \\
\hline $2007-2008$ & La Niña & Moderada \\
\hline $2009-2010$ & El Niño & Moderado \\
\hline $2010-2011$ & La Niña & Forte \\
\hline $2011-2012$ & La Niña & Fraca \\
\hline
\end{tabular}

Fonte: http://www.cpc.ncep.noaa.gov/products/analysis_ monitoring/ensostuff/ensoyears.shtml.

\section{RESULTADOS E DISCUSSÃO}

A precipitação acumulada anual para ambos os conjuntos preenchidos e homogeneizados apresentaram alta variabilidade em todo o período 
de estudo (Figura 3). A precipitação média calculada do conjunto de dados observados da EMA/NCINMET foi de $1294 \mathrm{~mm}$ (Figura 3a), sendo abaixo da precipitação obtida pelo conjunto EMA-TRMM (1571 mm) (Figura 3b) para a região de Seropédica, no período de 2000-2012.

Nota-se que acumulados de precipitação anual entre os anos de 2001 e 2003 (ciclo da La Niña-Fraca/ El Niño-Moderado) foram abaixo de $1200 \mathrm{~mm}$. Os anos de 2004 e 2007, anos estes que fazem parte do ciclo de El Niño-Fraco, não apresentaram grande variabilidade no regime. Os anos de 2003 e de 2008 a 2010 (ciclos de La Niña e El Niño-Moderado e La Niña Forte) (Tabela 2) foram considerados os anos mais chuvosos em relação à média do período em ambos os conjuntos de dados preenchidos, adotados no estudo (Figura 3).

Ocorreu grande variabilidade nos totais acumulados anuais de precipitação no período estudado, devendo-se isso à ocorrência dos ciclos de El Niño/La Niña, que interferem diretamente na atuação dos sistemas produtores de chuva (SFs, ZCAS e ZCOU) no Estado do Rio de Janeiro e, por consequência, no município de Seropédica. Entretanto, não podemos deixar de mencionar a influência de sistemas de mesoescala e locais, que contribuem para essa variabilidade no regime de precipitação. Verificou-se que os maiores totais acumulados anuais em ambos os conjuntos foram no mesmo período dos eventos de ENOS categorizados moderados (Figura 3), quando ocorreu um aumento considerável dos valores acumulados de precipitação. No entanto, para caracterizar esse padrão relacional, se faz necessário um período de no mínimo 30 e no máximo 60 anos de dados ininterruptos de precipitação devidamente preenchidos e homogeneizados, assim como a utilização de alguma ferramenta estatística, como, por exemplos, análise harmônica, técnica de wavelet ou análise multivariada (ACP).

Em relação aos sistemas produtores de chuva, sucederam-se mais episódios de ZCAS após o ano de 2003 (Tabela 1), devido à mudança da caracterização do fenômeno segundo a Climanálise. Mais de $80 \%$ dos eventos de ZCAS ocorrem com a presença de um cavado ou frente subtropical sobre o Oceano Atlântico na Região Sudeste. A banda de nebulosidade associada à ZCAS nem sempre aparece bem definida e homogênea. Esse comportamento é similar à ZCIT (Zona de Convergência Intertropical) ou aos SFs, significando que os sistemas podem estar presentes com pouca atividade. O fluxo de umidade em $850 \mathrm{hPa}$ aparece bem determinado, direcionado desde a Região Amazônica até o oceano, passando pelo Centro-Oeste e pelo Sudeste do Brasil. A Alta da Bolívia (AB) e o Cavado do Nordeste $(\mathrm{CNe})$, ou Vórtice do Nordeste (VNE), aparecem bem definidos apenas nos casos de ZCAS clássicas. Geralmente, a $\mathrm{AB}$ aparece, apesar de se apresentar desconfigurada em várias situações. $\mathrm{O}$ VNE pode não aparecer
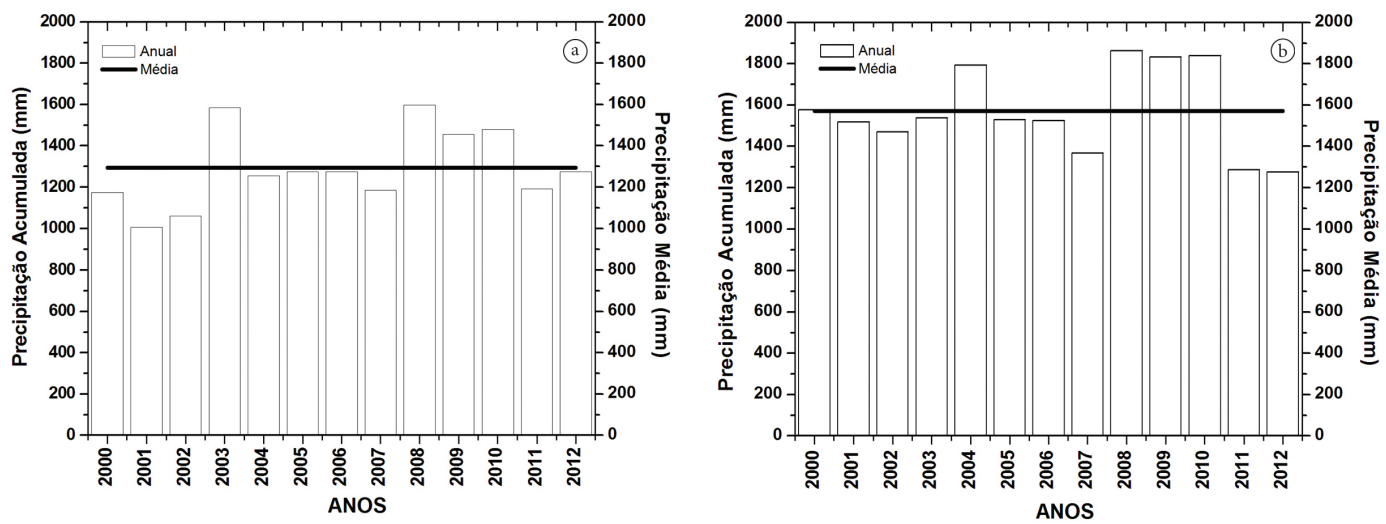

Figura 3. Precipitação média e anual acumulada $(\mathrm{mm})$ do EMA/NC - INMET (a) e do produto 3B43-TRMM /EMA (b) no município de Seropédica, no período de 2001-2012.

Figure 3. Average rainfall and annual accumulated (mm) of EMA / NC - INMET (a) and 3B43 TRMM / EMA product (b) on Seropédica at 2001-2012 period. 
em casos de ZCAS. A Zona de Convergência de Umidade em baixos níveis, provocada pela presença das características mencionadas anteriormente, deve persistir pelo menos por quatro dias para caracterizar a ZCAS. Será contabilizado como primeiro dia de ZCAS o momento em que se configurará o fenômeno. Se o processo durar menos de quatro dias, o evento será caracterizado como ZCOU.

Os eventos de SFs tiveram grande variabilidade no período estudado, tendo influenciado, principalmente, em anos considerados chuvosos, os quais, por sua vez, não configuraram um padrão relacional (Tabela 1). Segundo Fedorova \& Carvalho (2000), os SFs são observados com maior frequência em anos de El Niño do que em anos de La Niña, corroborando com os resultados encontrados para o Estado do Rio de Janeiro e, principalmente, influenciando na variabilidade temporal da chuva em Seropédica.

$\mathrm{Na}$ avaliação sazonal dos dados do EMA/ NC - INMET e EMA-TRMM, verificou-se que, na estação de verão (DJF), ocorreram os maiores valores de precipitação média acumulada - entre 180 e $250 \mathrm{~mm}(41,8$ e 47,8\%) (Figura 4a, b) -, enquanto que, na estação de outono (MAM), ocorreu uma diminuição considerável, correspondente a 24,6\% (EMA/NC - INMET) e 23\% (EMA-TRMM), com valores absolutos de 106 e $121 \mathrm{~mm}$. Isso é explicado pela não ocorrência de episódios de ZCAS/ZCOU, seguida de uma grande variabilidade na atuação de SFs (Oliveira, 1986) e, por fim, pela diminuição da atuação de sistemas de características locais (convecção local e circulação da brisa marítima/ terrestre), no município (Figura 4a, b).

No inverno (JJA), os valores médios de precipitação acumulada foram abaixo de $50 \mathrm{~mm}$ (EMA/NC - INMET e EMA-TRMM) com percentual de 9,7 e 6,6\%. Quando comparado com as demais estações, é mostrado claramente que esta é a estação com menor ocorrência de chuvas em Seropédica (Figura 4a, b). Os baixos valores de precipitação acumulada média se devem à variabilidade dos SFs e à não ocorrência de episódios de ZCAS/ZCOU, similares aos resultados obtidos anteriormente por Pereira \& Menezes (2004) e André et al. (2008).

A primavera (SON) volta a ter um aumento na precipitação acumulada média EMA/NC - INMET e EMA-TRMM na ordem de 103 e $118 \mathrm{~mm}$ $(23,9$ e 22,5\%). Em comparação às estações de inverno e outono, começam a se caracterizar padrões similares aos observados na estação de verão na região, como, por exemplo, o aumento da frequência dos sistemas sinóticos (SF, ZCOU e ZCAS), assim como fenômenos de mesoescala e locais citados anteriormente que atuam em Seropédica (Figura 4a, b).

Em geral, observou-se a influência marcante dos sistemas sinóticos, de mesoescala e locais, os quais,
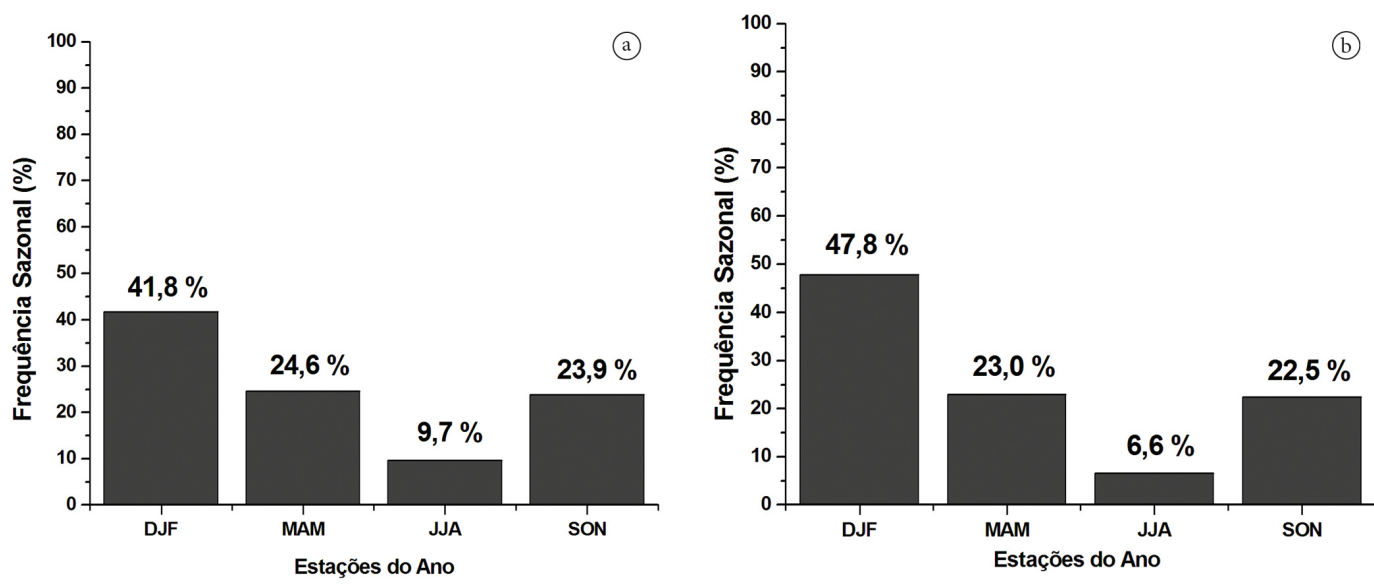

Figura 4. Frequência (\%) sazonal da precipitação do EMA/NC - INMET (a) e do produto 3B43-TRMM/EMA (b) para Seropédica, no período de 2000 a 2012.

Figure 4. Frequency (\%) seasonal precipitation EMA/NC - INMET (a) and 3B43 TRMM / EMA product (b) Seropédica at 2000-2012 period. 
por sua vez, interagem como terreno complexo que circunda a região de Seropédica (Figura 1) na distribuição do regime anual de precipitação. Sazonalmente, as estações de primavera e verão tiveram as maiores ocorrências percentuais de chuva em comparação às demais. Esse resultado foi condicionado pela in(desin)tensificação dos sistemas de mesoescala (brisa marítima/terrestre e brisa de vale/montanha) e locais (convecção local) atuantes na região nessas estações, associados ao relevo complexo, à proximidade da Baía de Sepetiba e ao fato de a região ser corredor de sistemas transientes, que resultam em elevada variabilidade temporal da precipitação em Seropédica. Os resultados obtidos por Moraes et al. (2005) mostram que o deslocamento das células convectivas obedece a duas principais trajetórias: Sudoeste - Nordeste (SFs e sistemas locais) e Norte - Sul (células convectivas originadas na região serrana que se deslocam em situações de circulações dominantes de Norte-Sul) e que corroboram com os resultados obtidos neste estudo. Foram encontrados maiores acumulados de precipitação, principalmente associados aos eventos de ENOS e a seu impacto nos sistemas sinóticos e locais. Preliminarmente, este estudo nos forneceu fortes indícios a respeito dos sistemas que caracterizam o regime de precipitação no município de Seropédica.

Na Tabela 3, foi observada correlação média sazonal entre o conjunto de dados (EMA/ NC - INMET) e o conjunto de dados (EMA-TRMM) de $r=0,64$ e $r^{2}=42 \%$. O EPE foi de $31 \mathrm{~mm}$ para os dados analisados e o índice de concordância $\mathrm{d}=0,49$. Apesar de o coeficiente de correlação ser satisfatório ( $r=0,64)$, no verão, o EPE foi maior, com 58,14 mm e índice $\mathrm{d}=0,35$, o que indica que o conjunto EMATRMM apresenta uma maior discrepância com os dados observados devido ao aumento dos processos convectivos nestes meses, com a maior ocorrência de episódios de ZCAS e ZCOU (Tabela 3).

No outono (MMA) (Tabela 3), a correlação (r) reduz-se para 0,55, assim como o EPE para 18,88 $\mathrm{mm}$ e o índice de concordância (d) aumenta para 0,48. Nesta estação, as ZCAS/ZCOU não atuam, apenas os SFs. No inverno (JJA), a concordância foi maior com $d=0,68$, em que o erro também foi menor, com 16,23 mm (Tabela 3). Nesta estação, há pouca atuação dos SFs no Estado do Rio de Janeiro.
Tabela 3. Análise estatística da precipitação sazonal (mm) da EMAS-NC (INMET) e os dados do satélite TRMM - EMAS para o município de Seropédica, no período de 2000 a 2012.

Table 3. Statistical analysis of seasonal precipitation $(\mathrm{mm})$ of EMAS-NC (INMET) and TRMM satellite - EMAS data for Seropédica municipality at 2000-2012 period.

\begin{tabular}{ccccc} 
Sazonalidade & $\mathbf{r}$ & $\mathbf{r}^{\mathbf{2}}$ & $\mathbf{E P E}$ & $\mathbf{d}$ \\
\hline DJF & 0,64 & 0,42 & 58,14 & 0,35 \\
MAM & 0,55 & 0,30 & 18,88 & 0,48 \\
\hline JJA & 056 & 0,31 & 16,23 & 0,68 \\
SON & 0,80 & 0,63 & 29,11 & 0,46 \\
Média & $\mathbf{0 , 6 4}$ & $\mathbf{0 , 4 2}$ & $\mathbf{3 0 , 5 9}$ & $\mathbf{0 , 4 9}$ \\
\hline
\end{tabular}

$\mathrm{Na}$ primavera (SON), a correlação (r) foi de 0,80 , sendo que o EPE foi o segundo menor entre os dados sazonais analisados, de $29,11 \mathrm{~mm}$, respectivamente. O índice de concordância (d) foi de 0,46 (Tabela 3). Nesta estação, os sistemas sinóticos começam dinamicamente a se caracterizar e se tornarem mais frequentes (SF, ZCOU e ZCAS).

Os resultados encontrados neste trabalho para o regime de precipitação sazonal no município de Seropédica não concordaram com os trabalhos realizados anteriormente por Nóbrega et al. (2008) e Collischonn et al. (2007), enquanto que o trabalho feito por Silva et al. (2013), comparando dados de EMS e os dados do satélite TRMM para o Vale Médio do Parapanema, em São Paulo-SP, apresentou também baixos valores de $\mathrm{r}^{2}$ e valores elevados do índice $\mathrm{d}$, similares aos resultados obtidos neste estudo. Os referidos autores comentaram que as diferenças encontradas podem estar associadas às escalas de cobertura (pontual e espacial), seguidas da resolução espacial do sensor do satélite TRMM de $0,25^{\circ}$.

\section{CONCLUSÕES}

O município de Seropédica apresenta totais acumulados anuais acima de $1000 \mathrm{~mm}$ em ambos os conjuntos de dados preenchidos e homogeneizados neste estudo, sendo esse padrão de chuva caracterizado por sistemas sinóticos e sistemas locais. Entretanto, não se descarta a influência do modo variabilidade ENOS, mesmo em um período curto de análise, visto que, na categorização de 
intensidade moderada do ENOS, são altos os totais anuais de precipitação acumulada.

As características identificadas ao longo desta avaliação mostraram que o município de Seropédica possui alta variabilidade nos padrões de precipitação, principalmente na escala interanual. A interação com o sistema local e sinótico, que ocorre ao longo do ano, principalmente nas estações de verão (maior percentual) e inverno (menor percentual), mostra-se com características bem definidas e distintas.

Os maiores acumulados de precipitação mensal identificados no estudo são observados em períodos de ocorrência conjunta de ZCAS/ZCOU e SFs, nos meses de novembro a janeiro no município de Seropédica, sendo que os menores acumulados são observados nos meses de maio a agosto.

Os dados do satélite TRMM são uma boa alternativa para o preenchimento de falhas e homogeneidade dos dados de precipitação em estações meteorológicas automáticas e convencionais, pois auxiliam na avaliação espacial e temporal de dados em regiões desprovidas de dados consistentes.

\section{AGRADECIMENTOS}

Os autores agradecem ao Instituto Nacional de Meteorologia (INMET), por ter cedido gentilmente os dados de precipitação. Ao Centro de Previsão de Tempo e Estudos Climáticos (CPTEC), e ao Instituto Nacional de Pesquisas Espaciais (INPE), pelos dados sinóticos.

\section{STATUS DA SUBMISSÃO}

Recebido: 20 ago., 2013

Aceito: 28 mar., 2014

Publicado: 30 jun., 2014

\section{AUTOR(ES) PARA CORRESPONDÊNCIA}

\section{José Francisco Oliveira Júnior}

Departamento de Ciências Ambientais - DCA, Universidade Federal Rural do Rio de Janeiro - UFRRJ, CEP 23890-000, Seropédica, RJ, Brasil

e-mail: jfoliverjr@lamma.ufrj.br

\section{REFERENNCIAS}

Allen RG, Jensen ME, Bornan RD. Operational estimates of reference evapotranspiration. Agronomy Journal 1989; 81(4): 650-662. http://dx.doi.org/10.2134/ agronj1989.00021962008100040019x

André RGB, Marques VS, Pinheiro FMA, Ferraudo AS. Identificação de regiões pluviometricamente homogêneas no estado do Rio de Janeiro, utilizando-se valores mensais. Revista Brasileira de Meteorologia 2008; 23(4): 501-509. http://dx.doi. org/10.1590/S0102-77862008000400009

Angelis CF, McGregor GR, Kidd C. A three year climatology of rainfall characteristics over tropical and subtropical South America based on TRMM-PR data. International Journal of Climatology 2004; 24(3): 385399. http://dx.doi.org/10.1002/joc.998

Cataldi M, Assad LPF, Torres Júnior AR, Alves JLD. Estudo da Influência das Anomalias da TSM do Atlântico Sul Extratropical na Região da Confluência Brasil Malvinas no Regime Hidrometeorológico de Verão do Sul e Sudeste do Brasil. Revista Brasileira de Meteorologia 2010; 25(4): 513-524. http://dx.doi. org/10.1590/S0102-77862010000400010

Carvalho DF, Silva DG, Souza AP, Gomes DP, Rocha HS. Coeficientes da equação de Angström-Prescott e sua influência na evapotranspiração de referência em Seropédica, RJ. Revista Brasileira de Engenharia Agrícola 2011; 15(8): 108-116.

Climanálise. Produtos Climanálise INPE/CPTEC. 2012. [cited 31 July 2012]. Available from: http://www.cptec. inpe.br/products/climanalise/.

Collischonn B, Allasia D, Collischonn W, Tucci CEM. Desempenho do satélite TRMM na estimativa de precipitação sobre a bacia do Paraguai superior. Revista Brasileira de Cartografia 2007; 59 (1): 93-99.

Dereczynski CP, Oliveira JS, Machado CO. Climatologia da Precipitação no Município do Rio de Janeiro. Revista Brasileira de Meteorologia 2009; 24 (1): 24-38. http:// dx.doi.org/10.1590/S0102-77862009000100003

Fedorova N, Carvalho MH. Processos Sinóticos em anos de La Niña e El Niño, Parte II: Zonas Frontais. Revista Brasileira de Meteorologia 2000, 15 (2): 57-72.

Huffman GJ, Adler RF, Bolvin DT, Gu G, Nelkin EJ, Bowman KP, et al. The TRMM Multi-satellite Precipitation Analysis (TMPA): Quasi-Global, Multiyear, Combined-Sensor Precipitation Estimates at Fine Scale. Journal of Hydrometeorology 2007; 8 (1): 3855. http://dx.doi.org/10.1175/JHM560.1

Jourdan P. Caracterização do Regime de Ventos Próximo à Superfície na Região Metropolitana do Rio de Janeiro [monografia]. Rio de Janeiro: Instituto de Geociências, 
Departamento de Meteorologia, Universidade Federal do Rio de Janeiro; 2007.

Gasparin KAC, Lyra GB, Francelino MR, Delgado RC, Oliveira-Júnior JF, Facco AG. Técnicas de geoprocessamento e sensoriamento remoto aplicadas à identificação de conflitos do uso da terra em Seropédica. Floresta e Ambiente 2013; 20 (3). http:// dx.doi.org/10.4322/floram.2013.030

Kummerow C, William B, Toshiaki K, James S, Joanne $\mathrm{S}$. The tropical rainfall measuring mission (TRMM) sensor package. Journal of Atmospheric and Oceanic Technology 1998; 15(3): 809-817. http://dx.doi. org/10.1175/1520-0426(1998)015\%3C0809:TTRMMT \%3E2.0.CO;2

Kummerow C, Simpson J, Thiele O, Barnes W, Chang ATC, Stocker E, et al. The Status of the Tropical Rainfall Measuring Mission (TRMM) after Two Years in Orbit. Journal of Applied Meteorology 2000; 39(1): 1965-1982. http://dx.doi.org/10.1175/1520-0450(2001)040\%3C196 5:TSOTTR\%3E2.0.CO;2

Lyra GB, Garcia BIL, Piedade SMS, Sediyama GC, Sentelhas PC. Regiões homogêneas e funções de distribuição de probabilidade da precipitação pluvial no Estado de Táchira, Venezuela. Pesquisa Agropecuária Brasileira 2006; 41(2): 205-215. http://dx.doi. org/10.1590/S0100-204X2006000200004

Moraes NO, Marton E, Pimentel LCG. Simulações Numéricas da Formação de Ilha de Calor na Região Metropolitana do Rio de Janeiro. Anuário do Instituto de Geociências - UFRJ 2005; 28(2):116-138.

Nóbrega RS, De Souza ÊP, Galvíncio JD. Análise da Estimativa de Precipitação do TRMM na SubBacia da Amazônia Ocidental. Revista de Geografia (Recife) 2008; 25(1): 06-20.

Oliveira AS. Interações entre sistemas frontais na América do Sul e a convecção da Amazônia [dissertação]. São José dos Campos: Instituto Nacional de Pesquisas Espaciais; 1986.

Oliveira-Júnior JF. Estudo da Camada Limite Atmosférica na Região de Angra dos Reis através do Modelo de Mesoescala MM5 e Dados Observacionais [tese]. Rio de Janeiro: Universidade Federal do Rio de Janeiro; 2008. http://dx.doi.org/10.4322/floram.2012.011
Oliveira-Júnior JF, Lyra GB, Gois G, Brito TT, Moura NSH.Análise dehomogeneidadedesériespluviométricas para determinação do índice de seca IPP no estado de Alagoas. Floresta e Ambiente 2012; 19(1): 101-112.

Pereira TRA, Menezes WF. Estudo Preliminar da Frequência de Sistemas Sinóticos que Atingem o Estado do Rio de Janeiro. In: XIII Congresso Brasileiro de Meteorologia; 2004; Fortaleza. Ceára: Sociedade Brasileira de Meteorologia; 2004.

Reboita MS, Gan MA, Rocha RP, Ambrizzi T. Regimes de Precipitação na América do Sul: Uma Revisão Bibliográfica. Revista Brasileira de Meteorologia 2010; 25(2): 185-204. http://dx.doi. org/10.1590/S0102-77862010000200004

Satyamurty P, Nobre CA, Silva Dias PL. Tropics-South America. Chapterin Meteorology of the Southern Hemisphere. Meteorological Monograph - American Metetorology Society 1998; 27(1): 201-225.

Silva DF, Pantano AP, Camargo MBP. Análise de dados de precipitação estimados pelo satélite TRMM para o vale do médio Parapanema - SP. Revista Engenharia na Agricultura 2013; 21(2): 138-147. http://dx.doi. org/10.13083/1414-3984.v21n02a04

Trenberth KE. The Definition of El Niño. Bulletin of the American Meteorological Society 1997; 78(12): 2771-2777. http://dx.doi.org/10.1175/15200477(1997)078\%3C2771:TDOENO\%3E2.0.CO;2

Wanderley HS, Sediyama GC, Justino FB, Alencar LP, Delgado RC. Variabilidade da precipitação no Sertão do São Francisco, estado de Alagoas. Revista Brasileira de Engenharia Agrícola e Ambiental 2013; 17(7): 790-795. http://dx.doi.org/10.1590/S1415-43662013000700014

Willmott CJ, ACkleson SG, Davis JJ, Feddema JJ, Klink KM, Legates DR, et al. Statistics for the evaluation and comparison of models. Journal of Geography Research 1985; 90(5): 8995-9005.

Zeri M, Oliveira-Júnior JF, Lyra GB. Spatiotemporal analysis of particulate matter, sulfur dioxide and carbon monoxide concentrations over the city of Rio de Janeiro, Brazil. Meteorology and Atmospheric Physics 2011; 113(3): 1-14. 